\title{
Capture, unfolding, and detection of individual tRNA molecules using a nanopore device
}

\author{
Andrew M. Smith ${ }^{1,2}$, Robin Abu-Shumays ${ }^{2}$, Mark Akeson $^{2,3}$ and David L. Bernick ${ }^{2 *}$ \\ ${ }^{1}$ Department of Chemistry and Biochemistry, University of California Santa Cruz, Santa Cruz, CA, USA, ${ }^{2}$ Department of \\ Biomolecular Engineering, University of California Santa Cruz, Santa Cruz, CA, USA, ${ }^{3}$ Genomics Institute, University of California \\ Santa Cruz, Santa Cruz, CA, USA
}

OPEN ACCESS

Edited by:

Nikola Kneževic

European University, Serbia

Reviewed by:

Samir M. labal,

Purdue University, USA

Rebecca Medda

University of Heidelberg, Germany

${ }^{*}$ Correspondence:

David L. Bernick,

Nanopore Research Group,

Department of Biomolecular

Engineering, University of California Santa Cruz, 1156 High Street, Santa

Cruz, CA 95060, USA

dbernick@soe.ucsc.edu

Specialty section:

This article was submitted to

Nanobiotechnology, a section of the journal Frontiers in Bioengineering and Biotechnology

Received: 11 April 2015 Accepted: 10 June 2015

Published: 24 June 2015

Citation:

Smith AM, Abu-Shumays $R$, Akeson M and Bernick DL (2015)

Capture, unfolding, and detection of individual tRNA molecules using a nanopore device. Front. Bioeng. Biotechnol. 3:91. doi: 10.3389/fbioe.2015.00091
Transfer RNAs (tRNA) are the most common RNA molecules in cells and have critical roles as both translators of the genetic code and regulators of protein synthesis. As such, numerous methods have focused on studying tRNA abundance and regulation, with the most widely used methods being RNA-seq and microarrays. Though revolutionary to transcriptomics, these assays are limited by an inability to encode tRNA modifications in the requisite cDNA. These modifications are abundant in tRNA and critical to their function. Here, we describe proof-of-concept experiments where individual tRNA molecules are examined as linear strands using a biological nanopore. This method utilizes an enzymatically ligated synthetic DNA adapter to concentrate tRNA at the lipid bilayer of the nanopore device and efficiently denature individual tRNA molecules, as they are pulled through the $\alpha$-hemolysin $(\alpha-H L)$ nanopore. Additionally, the DNA adapter provides a loading site for $\phi 29$ DNA polymerase ( $\$ 29$ DNAP), which acts as a brake on the translocating tRNA. This increases the dwell time of adapted tRNA in the nanopore, allowing us to identify the region of the nanopore signal that is produced by the translocating tRNA itself. Using adapter-modified Escherichia coli tRNA ${ }^{\text {fMet }}$ and tRNA ${ }^{\text {Lys }}$, we show that the nanopore signal during controlled translocation is dependent on the identity of the tRNA. This confirms that adapter-modified tRNA can translocate end-toend through nanopores and provide the foundation for future work in direct sequencing of individual transfer RNA with a nanopore-based device.

Keywords: tRNA, nanopore, nanotechnology, RNA sequencing, single molecule

\section{Introduction}

Transfer RNAs (tRNA) decode genetic information, delivering to the protein-synthesizing ribosome the individual amino acids specified by each codon of a messenger RNA. In light of this, it is unsurprising that they are the most numerous RNA species in the cell, composing approximately $80 \%$ of the RNA molecules per generation in yeast (Phizicky and Hopper, 2010). These small, non-coding RNA molecules contain numerous post-transcriptionally modified nucleotides, which contribute to tRNA fold stabilization, codon recognition, and aminoacylation [reviewed in Gustilo et al. (2008) and Motorin and Helm (2010)]. A growing body of research indicates that tRNAs and their nucleotide modifications are directly targeted for regulation as well as acting as potential regulators themselves [reviewed in Yi and Pan (2011) and Raina and Ibba (2014)]. 
Current methods for analysis of tRNAs, and RNA in general, include RNA-seq, microarray, and mass spectrometry. These methods are proven tools for detection of novel tRNAs and global tRNA expression patterns (Dittmar et al., 2006; Chan et al., 2011). However, each method has limitations.

High-throughput RNA sequencing (RNA-Seq) methods require extensive library preparation, including PCR amplification, to prepare cellular RNA for sequencing. A reverse transcription (RT) step is necessary to copy the original RNA sequence to cDNA, which results in loss of the original RNA strand. Additionally, the RT step is impeded by the occurrence of structure and nucleotide modifications, which are both commonly found in tRNAs. These "RT-stops" result in truncated cDNA. While these RT stops have been exploited by various methods to infer structure or nucleotide modification state in RNA, they do not permit direct detection of nucleotide sequence and modification identity along intact RNA (Omer et al., 2000; Merino et al., 2005; Bernick et al., 2012; Vilfan et al., 2013). Methods have been developed for detecting a few specific RNA modifications, such as bisulfite treatment for 5-methylcytosine or immunoprecipitation for N6-methyladenine, but these require additional labor-intensive steps (Schaefer et al., 2009; Dominissini et al., 2012).

Microarrays share a similar problem with loss of modification information and extended processing times. Success has been reported in detecting nucleotide modifications in tRNAs that affect hybridization of the target molecule to array probes, which limits detection to those modifications that affect Watson-Crick base-pairing (Hiley et al., 2005; Saikia et al., 2010). In addition, microarray probe design requires prior knowledge of the target sequence, which limits detection to known RNAs.

Liquid chromatography coupled to mass spectrometry (LCMS) has also been applied to study tRNAs. In particular, two recent publications have used LC-MS to examine dynamic changes in tRNA modification state in Saccharomyces cerevisiae under varied environmental growth conditions (Chan et al., 2010, 2012). However, the nucleolytic fragmentation that is required by this method prevents observing modifications in the full sequence context of the tRNA.

Detection of tRNA subpopulations that result from variability in modification, as seen in mitochondrial diseases, will benefit from direct interrogation of intact, single tRNA molecules (Suzuki et al., 2011). In two specific mitochondrial examples, failed tRNA modifications cause dysfunctional translation of mitochondrial enzymes. These in turn result in the mitochondrial encephalopathies, myoclonic epilepsy with ragged-red fibers (MERRF), and mitochondrial encephalomyopathy, lactic acidosis, and stroke-like episodes (MELAS) (Yasukawa et al., 2001; Kirino et al., 2004). In these examples, a method that examines individual tRNA molecules could reveal the extent of incompletely modified or mutant forms in these complex disease phenotypes.

Nanopore sensors interrogate single molecules and should allow for examination of several thousand individual tRNA in a single experiment. Nearly 20 years ago, biological nanopores with 1-2 nm limiting apertures were conceived as single-molecule sensors for nucleic acids (Kasianowicz et al., 1996). In concept, the nucleotide sequence of an individual molecule would be read by observing changes in ionic current as the linearized strand is electrophoresed through the nanopore aperture. Recent developments in sensing DNA have coupled an enzyme to regulate DNA movement in single nucleotide steps through a nanopore, which produced ionic current traces that provide a single base readout of DNA sequence (Cherf et al., 2012; Manrao et al., 2012). Additionally, experiments by our group and others have shown that DNA cytosine modifications can be detected with high confidence from individual nanopore reads of chemically synthesized DNA (Laszlo et al., 2013; Schreiber et al., 2013). Applying these principles should provide a high-throughput method to directly examine tRNA nucleotide sequence.

With RNA, strand sequencing with single-nucleotide resolution has yet to be demonstrated with a nanopore device. However, Ayub and Bayley have shown that immobilized RNA strands within a modified $\alpha$-HL nanopore apparatus yield distinct ionic current amplitudes that discriminate between canonical and select modified ribonucleotides (Ayub and Bayley, 2012). Further, solidstate nanopores with pore-sizes that exceed $3 \mathrm{~nm}$ have been shown to distinguish folded tRNA molecules from linear doublestranded RNA and DNA (Wanunu et al., 2010). By extension, these results suggest that nanopore sensors could detect submolecular features of tRNA, including nucleotide modifications, if tRNAs can be mechanically unfolded and electrically motivated to pass through the pore.

With this in mind, we sought to develop a mechanism to specifically capture tRNA molecules, promote their mechanical unfolding, and initiate threading of the linearized strand through the nanopore lumen. To this end, we designed an oligonucleotide adapter that can be attached to intact tRNAs. To slow tRNA translocation through the pore, we employed a non-catalytic protein "brake" that loads onto the adapter. This allowed us to determine the direction of strand translocation and provided sufficient resolution to determine ionic current signal features associated with the translocating adapter and the tRNA. Results presented here demonstrate that tRNA attached to such an adapter and modulated by a protein brake can be completely translocated through the $\alpha$-HL nanopore, and that Escherichia coli $\mathrm{tRNA}^{\mathrm{fMet}}$ and $\mathrm{tRNA}^{\text {Lys }}$ produce differentiable nanopore signals in this system. This provides the foundation for future work aimed at achieving single nucleotide resolution of individual transfer RNA.

\section{Materials and Methods}

\section{Oligonucleotide Synthesis and Purification}

All oligonucleotides were synthesized by the Stanford Protein and Nucleic Acids facility (PAN) using standard phosphoramidite chemistry. Oligonucleotides were purified by denaturing $7 \mathrm{M}$ urea polyacrylamide gel electrophoresis (PAGE) in $1 \times \mathrm{TBE}$, followed by overnight elution from an excised band at $4^{\circ} \mathrm{C}$ in $300 \mathrm{mM}$ Sodium Acetate pH 5.2 and $1 \mathrm{mM}$ EDTA. DNA was precipitated and recovered by adding $100 \%$ molecular biology grade ethanol (Sigma Aldrich) to $70 \%$ final v/v and centrifuged for $30 \mathrm{~min}$ at $14,000 \times g$ and $4^{\circ} \mathrm{C}$. Alternately, RNA-containing oligonucleotides were recovered by precipitation in $75 \%$ ethanol $\mathrm{v} / \mathrm{v}$ and centrifuged at $14,000 \times g$ for $30 \mathrm{~min}$ at $4^{\circ} \mathrm{C}$. The ethanol 
mixture was aspirated and oligonucleotides were then washed with an equal volume of 70 or $75 \%$ ethanol and pelleted again for $10 \mathrm{~min}$ at $14,000 \times \mathrm{g}$ and $4^{\circ} \mathrm{C}$. Ethanol was aspirated and the pellets were allowed to dry under vacuum to remove residual ethanol. Oligonucleotides were then resuspended in nuclease free water, quantified by Nanodrop (Thermo Scientific), and stored at $-80^{\circ} \mathrm{C}$.

\section{Adapter Design and Hybridization}

The two oligonucleotides of the tRNA adapter were designed to form a partially double-stranded $y$-shape. The design included one end that targeted the ACCA tail of tRNA, while the other end contained unpaired single stranded regions (see Figure 1A). The adapter strands also included positions where abasic $\left(1^{\prime}-\mathrm{H}\right.$ deoxyribose) residues were incorporated to act as ionic current markers for the different ends of the adapted RNA molecule.

$5^{\prime}$ leading strand oligonucleotide (bolded italicized bases indicate RNA; plain letters are DNA, $\mathrm{X}$ indicates an abasic $1^{\prime}$ $\mathrm{H}$ deoxyribose): $5^{\prime}$-CTCACCTATCCTTCCACTCATACTATCA TTATCTXTCAGATCTCACTAUCUGGU-3'.

$3^{\prime}$ trailing strand oligonucleotide ( $\mathrm{X}$ indicates an abasic $1^{\prime}$ $\mathrm{H}$ deoxyribose; $\mathrm{Z}$ is a triethylene glycol-cholesterol): $5^{\prime}$-phosGATXGTGAGATCTGATTTTTTTTTTTTTTTZ-3'.

For experiments to determine which end of the adapted RNA molecules entered the pore first, new adapter nucleotides were synthesized with one of the abasic markers (as indicated above) replaced by the nucleotide that restored complementarity in the double stranded portion of the adapter. In these experiments, one of the following oligonucleotides replaced its corresponding abasic-containing strand in the adapter:

$5^{\prime}$ leading strand oligonucleotide: $5^{\prime}$-CTCACCTATCCTTCCA CTCATACTATCATTATCTCTCAGATCTCACTAUCUGGU $-3^{\prime}$.

$3^{\prime}$ trailing strand oligonucleotide: $5^{\prime}$-phos-GATAGTGAGATC TGATTTTTTTTTTTTTTTZ- ${ }^{\prime}$.

Adapters were formed by combining leading and trailing strands at $100 \mu \mathrm{M}$ in $10 \mathrm{mM}$ Tris- $\mathrm{HCl} \mathrm{pH} 8$ and $50 \mathrm{mM} \mathrm{NaCl}$. The mixture was heated to $95^{\circ} \mathrm{C}$ for $30 \mathrm{~s}$ and allowed to cool to room temperature.

\section{RNA, RNA Ligation Reaction, and Purification of Full-Length Products}

Escherichia coli $\mathrm{tRNA}^{\mathrm{Lys}}$ and $\mathrm{tRNA}^{\mathrm{fMet}}$ were purchased from Sigma-Aldrich. The RNA hairpin control was prepared by the Stanford PAN facility using the sequence $5^{\prime}$-phosCGCGGGGUUUUUCCCCGCAACCA- ${ }^{\prime}$. RNA substrates were ligated to adapters using RNA Ligase 2 (NEB). Ligation reactions were carried out in $20 \mu \mathrm{L}$ of buffer recommended by the manufacturer supplemented with $0.5 \mathrm{mM}$ ATP, 5\% PEG 8000 , and $2 \mu \mathrm{M}$ each of RNA and adapter. To end the reaction and prepare the sample for purification, $50 \mu \mathrm{L}$ urea loading buffer $(7 \mathrm{M}$ urea and $0.1 \times \mathrm{TBE})$ was added to the sample and heated to $95^{\circ} \mathrm{C}$ for $5 \mathrm{~min}$. The products were separated on a $7 \mathrm{M}$ urea PAGE gel in $1 \times$ TBE. The gel was post-stained with $2 \times$ SybrGold (Life Technologies) and product of appropriate size for the complete ligation product was excised. Ligated RNA was recovered by electroelution into $3.5 \mathrm{kDa}$ MWCO D-tubes (Novagen) at $100 \mathrm{~V}$ for $2 \mathrm{~h}$ in $1 \times$ TBE. Recovered ligated RNA

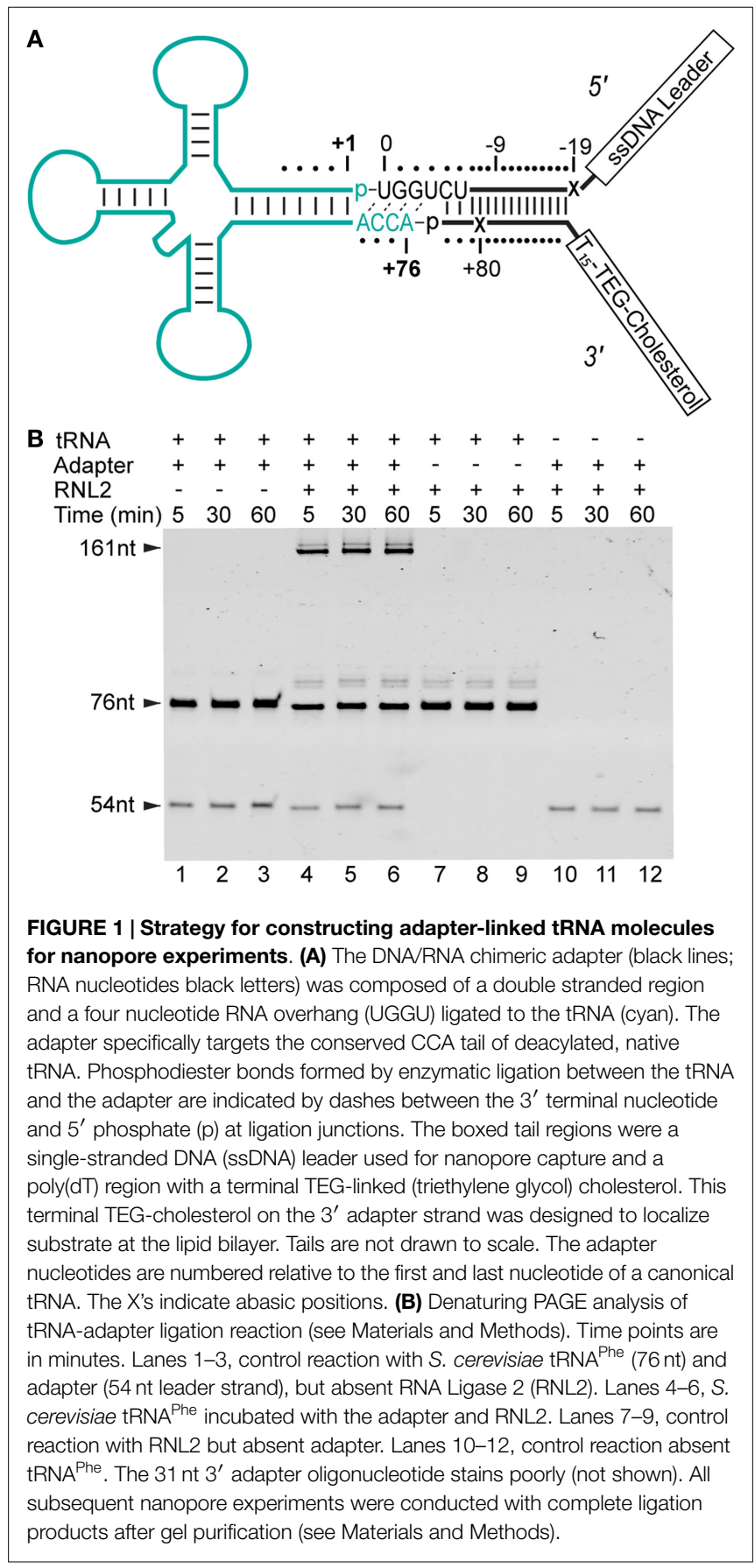

preparations were ethanol precipitated, quantified by Nanodrop (Thermo Scientific), and stored at $-80^{\circ} \mathrm{C}$.

\section{Nanopore Experiments}

Nanopore experiments were performed using a single $\alpha$-HL nanopore (indicated by a step-wise increase in ionic current, range 68.0-72.5 pA) embedded in a planar 1,2-diphytanoyl-synglycero-phosphatidylcholine bilayer established on a $\sim 25 \mu \mathrm{M}$ aperture, as described previously (Akeson et al., 1999). Experiments were conducted in $0.3 \mathrm{M} \mathrm{KCl}$ and $10 \mathrm{mM}$ HEPES $\mathrm{pH} 8.0$ at $28^{\circ} \mathrm{C}\left( \pm 0.4^{\circ} \mathrm{C}\right)$ at $180 \mathrm{mV}$ (trans well positive). Dithiothreitol 
(2 mM final) and EDTA pH 8.0 ( $1 \mathrm{mM}$ final) were added to the well on the cis side of the bilayer. In the case of experiments looking at the effect of $\mathrm{Mg}^{2+}, \mathrm{MgCl}_{2}$ was added to the buffer to $5 \mathrm{mM}$ and EDTA was omitted from the cis side well. Nucleic acid substrates were added to $0.5 \mathrm{nM}$, unless otherwise noted to the cis well, and allowed to incubate $2 \mathrm{~min}$ to associate with the bilayer. For experiments where $\phi 29$ DNAP was to be added, an additional $12.5 \mathrm{~min}$ incubation was allowed; during this period, we observed captures of unbound RNA substrate prior to adding \$29 DNAP (Enzymatics) to $75 \mathrm{nM}$. Ionic current measurements were collected with an Axon Axopatch 200B (Molecular Devices) patch-clamp amplifier in whole-cell, voltage-clamped mode, and filtered with an analog low-pass Bessel filter at $5 \mathrm{kHz}$, then digitized using an Axon Digidata 1440A analog-to-digital converter (Molecular Devices) at $100 \mathrm{kHz}$ bandwidth.

\section{Event Detection and Ionic Current Region Measurements}

For nanopore experiments that examined populations of ionic current blockade events, a custom developed event detection program was used (https://github.com/jmschrei/PyPore). The detection algorithm identified ionic current blockades that were self-terminating by selecting for segments that deflected from open nanopore current (68.0-72.5 pA) below a cutoff of 45 or $55 \mathrm{pA}$ and with a minimum duration of $>0.1 \mathrm{~ms}$, where voltage was not reversed to eject the stand from the pore (current never $<0 \mathrm{pA}$ ). For experiments where individual ionic current blockade events were examined, raw nanopore ionic current data were filtered with a digital $2 \mathrm{kHz}$ low-pass Bessel filter and analyzed using Clampfit 10.4 (Molecular Devices). For $\phi 29$ DNAP molecular braking experiments, events were selected from current blockades that had durations $>1 \mathrm{~s}$ and self-terminated. Event classification from adapted RNA(hairpin) substrates were analyzed as articulated in the text. For adapted tRNA data, complete translocation events were selected based on the criteria that they contained exactly two abasic-dependent regions; these were defined as high current regions with a mean current $>33.5 \mathrm{pA},<36.5 \mathrm{pA}$, and minimum a duration $>2 \mathrm{~ms}$. After selection as complete translocation events, duration and mean current for states I-III were measured by hand using Clampfit's internal statistics measuring program.

\section{Semi-Logarithmic Decision Boundary and Accuracy Derivation}

Event durations were transformed to log-durations $\left(\log _{10}\right)$ and a linear decision boundary was established using the kernlab (v 0.9-19) package (Karatzoglou et al., 2004) under R (v 3.0.2). The ksvm parameters used were "type = 'C-svc', kernel = 'vanilladot,' $C=10$ ” to produce a soft-margin decision boundary. To assess classification accuracy, a fivefold training/test regimen was used. The data set was shuffled and then partitioned into five groups of nearly equal size. In a series of five tests, one of the five groups was withheld as a test set, while the decision boundary was calculated using the remaining four. This was repeated for each of the five groups. This procedure was repeated 50 times, providing 250 assessments of accuracy. Mean and standard deviation of these 250 accuracy scores are reported. For this study, we used a balanced accuracy score, calculated as the mean recall rate for each of the two data classes. For two classes with labels $\{1,-1\}$,

$$
\text { balanced accuracy }=\frac{\frac{\operatorname{pred}(1)}{\operatorname{true}(1)}+\frac{\operatorname{pred}(-1)}{\operatorname{true}(-1)}}{2}
$$

where $\operatorname{true}(n)$ are counts of test data labeled $n$, and $\operatorname{pred}(n)$ are counts of test data that are correctly classified. The graphic provided in Figure 6 was derived using the full dataset and the kernlab package with parameters as above. Margins (dotted lines) in Figure 6 provide the optimized bounds that maximize the proper classification of labeled data outside the margin while minimizing the cost of misclassified data on the "wrong" side of that margin (Cortes and Vapnik, 1995).

\section{Results}

\section{Capture and Threading of tRNA Through the $\alpha-H L$ Nanopore is Facilitated by Ligation of an Oligonucleotide Adapter to the tRNA}

Reading the nucleotide composition of individual tRNA molecules will require capture, denaturation, and threading of each strand sequentially through the nanopore. In our initial experiments, we found that native tRNA molecules caused long $(>30 \mathrm{~s}$ ) ionic current blockades of the $\alpha$-HL pore (data not shown). These molecules had to be ejected by voltage reversal to re-establish an open-pore ionic current. This suggested that tRNA molecules in their native form would not readily translocate through the $\alpha$-HL nanopore.

We reasoned that an extended single-stranded region, longer than the ACCA in native tRNA, may be needed to initiate threading of each tRNA molecule into the lumen of $\alpha$-HL. To accomplish this, we devised a strategy to covalently attach synthetic nucleic acid strands to the $3^{\prime}$ and $5^{\prime}$ ends of the tRNA. This was achieved using a $Y$-shaped, partially double-stranded DNA-RNA adapter that contained a $3^{\prime}$ RNA overhang complementary to the universally conserved CCA tail in tRNA (Figure 1A). The strand of the adapter, which bares the $3^{\prime}$ RNA overhang, was designed to be ligated to the 5 ' end of a tRNA (referred to as the "leading strand"). The unpaired region of the leading strand contained 35 singlestranded nucleotides and was designed to facilitate capture and threading into the nanopore. The double-stranded region of the adapter was designed to allow a dsRNA ligation, and effectively extended the tRNA adapter stem by 15 base-pairs.

The strand of the adapter that was designed to be covalently attached to the $3^{\prime}$ end of the tRNA (referred to as the "trailing strand") incorporated a cholesterol tag at its $3^{\prime}$ end. This was designed to locally concentrate the adapted tRNA at the lipid bilayer-aqueous interface of the nanopore experimental setup. Association of the cholesterol moiety on the $3^{\prime}$ trailing strand with the bilayer should favor capture of the free $5^{\prime}$ end of the leading strand in the electric field surrounding the nanopore. Finally, the adapter design incorporated abasic residues into both leading and trailing strands to act as ionic current signal markers upstream and downstream of the ligated tRNA.

We initially tested if the adapter could be enzymatically ligated to tRNA using T4 RNA Ligase 2 (RNL2). Analysis of that ligation 
reaction revealed that a product of appropriate size $(\sim 160 \mathrm{nt})$ was generated only in the presence of both the adapter and a model tRNA substrate ( $S$. cerevisiae tRNA $^{\text {Phe }}$ ) (Figure 1B). These results indicated that enzymatic ligation with RNL2 was an effective method for adding adapter strands to this model tRNA. To test this further, we ran additional ligation experiments with $E$. coli $\mathrm{tRNA}^{\mathrm{fMet}}$. This tRNA represented a more challenging substrate because it contains a non-canonical nucleotide pair at the end of the acceptor stem (RajBhandary, 1994). Our results showed that E. coli $\mathrm{tRNA}^{\mathrm{fMet}}$ was also a reactive substrate for ligation to the adapter (data not shown).

Initial nanopore experiments were performed with adapted E. coli tRNA $^{\text {fMet }}$ using an established single-channel apparatus (see Materials and Methods) (Figure 2A). Ionic current blockade events were observed with a typical duration of tens of milliseconds (mean duration $=10^{-2.6 \pm 0.06} \mathrm{~s}$, variation of the mean shown as SEM) (Figures 2B,C, magenta circles). These events were longer than the events observed for the adapter alone, which were typically a millisecond or less (mean duration $=10^{-3.9 \pm 0.04} \mathrm{~s}$ ) (Figure 2C, open triangles). The increased duration of events with adapted $\mathrm{tRNA}{ }^{\mathrm{fMet}}$ suggested that longer or more structured molecules were being captured and translocated through the pore. The extremely short duration blockade events observed with the adapter alone were consistent with single-stranded nucleic acids being translocated through the pore (Deamer and Branton, 2002).
If in fact the longer dwell time was caused by tRNA, then conditions known to stabilize tRNA should increase event duration further. Magnesium ions are known to stabilize the tertiary fold of tRNA (Stein and Crothers, 1976; Serebrov et al., 2001). When we added magnesium chloride to the experimental buffer (5 mM final concentration), we observed an increase in event duration (100-fold or $2 \log _{10}$ units) relative to experiments absent $\mathrm{Mg}^{2+}$ (mean duration $=10^{-1.4 \pm 0.1} \mathrm{~s}$ ) (Figure 2C, blue squares). These blockades were self-terminating, as seen in experiments without $\mathrm{Mg}^{2+}$. This result was consistent with capture of $\mathrm{Mg}^{2+}$ stabilized tRNA. Together, these results suggested that the electricfield driven denaturation of secondary, and potentially tertiary structure facilitated the translocation of tRNA through the pore.

\section{$\phi 29$ DNAP Acts as a Molecular Brake During Translocation of Adapted RNA Under Non-Catalytic Conditions}

In previous studies with both RNA and DNA, uncontrolled polynucleotide translocation rate through a nanopore was too high to resolve single nucleotide-level information about the translocating strand (Deamer and Branton, 2002). Therefore, we sought to slow the translocation of adapted tRNA to improve resolution of tRNA features and to provide definitive evidence that adapted tRNA molecules transit the pore in their entirety. Control of DNA transit rates using DNA polymerases has been documented, and Lieberman et al. (2010) showed that $\phi 29$ DNAP
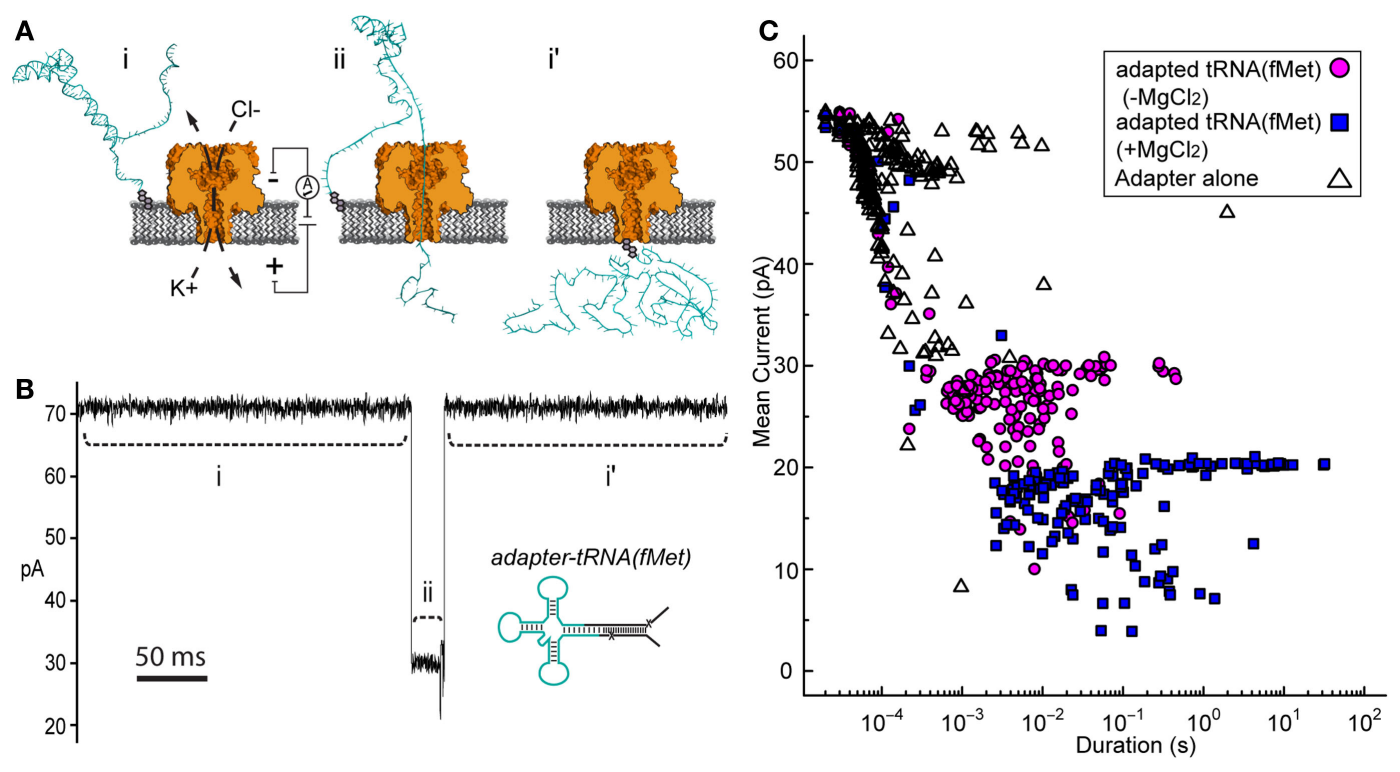

FIGURE 2 | Adapted tRNA-dependent ionic current blockades observed during single-channel $\alpha-\mathrm{HL}$ nanopore experiments. (A) Cartoon illustration of the single-channel nanopore apparatus and a proposed adapted tRNA translocation event. (i) A constant voltage (trans side + ) is applied across a single $\alpha-H L$ nanopore (orange) embedded in a lipid bilayer (gray). (ii) Electrophoretic capture of an adapted tRNA (cyan) results in a decrease in the measured ionic current through the nanopore. (i') Return to open channel current when the tRNA clears the pore in the trans compartment. (B) An ionic current trace from a nanopore experiment with adapted $E$. coli $\mathrm{RRNA}^{\mathrm{fMet}}$ (inset). lonic current regions i-ii and i' in the trace (dashed lines) correspond to the proposed tRNA translocation event in (A). The blockade event shown is typical of thousands of events observed during nanopore experiments with adapted tRNA ${ }^{\text {fMet }}$. Scale bar indicates $50 \mathrm{~ms}$. (C) Nanopore blockade mean ionic current versus duration caused by adapted tRNA ${ }^{\mathrm{fMet}}$ in the presence or absence of $\mathrm{Mg}^{2+}$. The mean current and duration of approximately 200 events are shown for representative nanopore experiments with either adapted tRNA ${ }^{\mathrm{fMet}}\left(-\mathrm{Mg}^{2+}\right)$ (magenta circles) or adapted tRNA ${ }^{\mathrm{fMet}}\left(+\mathrm{Mg}^{2+}\right)$ (blue squares). The adapter on its own $\left(-\mathrm{Mg}^{2+}\right)$ (open triangles) was also examined as a negative control. In all cases, single-channel $\alpha-\mathrm{HL}$ nanopore experiments were conducted at $180 \mathrm{mV}$ (trans side+) with tRNA substrate at $0.5 \mathrm{nM}$ in $0.3 \mathrm{M} \mathrm{KCl}, 10 \mathrm{mM}$ HEPES (pH 8.0), and $\pm 5 \mathrm{mM} \mathrm{MgCl}_{2}$ (see Materials and Methods). 
can serve as a "molecular brake" that controls the rate of DNA translocation through the $\alpha$-HL pore under non-catalytic conditions (absent $\mathrm{Mg}^{2+}$ and dNTPs) (Lieberman et al., 2010). Furthermore, this molecular brake activity of $\phi 29$ DNAP has been observed on a chimeric DNA-RNA substrate with a nanopore device (J. Clarke, Oxford Nanopore Technologies, personal communication).

We wanted to determine if the $\$ 29$ DNAP molecular brake could also be used to control translocation of RNA molecules containing more complex structures, such as stem-loops found in tRNA. For this, we synthesized a simple RNA hairpin, which we ligated to the tRNA adapter (Figure 3A). This synthetic RNA [referred to as RNA(hairpin)] mimicked the acceptor stem of tRNA $^{\text {fMet }}$, where the two halves of the acceptor stem were linked by a short loop region of five uracil residues.

Nanopore capture of complexes formed between the adapted RNA and $\phi 29$ DNAP, similar to those seen by Lieberman et al., should result in greatly increased dwell time of individual adapted RNA molecules within the aperture of $\alpha$-HL. This would be observed as population of longer duration nanopore current blockades, which would be distinct from the shorter duration events of unbound adapted RNA strands.

Control nanopore experiments with the adapted RNA(hairpin) construct absent $\$ 29$ DNAP resulted in current blockade events with mean duration on the order of milliseconds (mean duration $=10^{-3.2 \pm 0.02} \mathrm{~s}$ ) (Figure 3C, magenta circles). Addition of $\phi 29$ DNAP to the buffer solution containing adapted $\mathrm{RNA}$ (hairpin) on the cis side of the nanopore apparatus produced two different types of current blockade events. These events typically fell into one of two populations: a short duration population (duration $<0.1 \mathrm{~s}$, mean $10^{-2.9 \pm 0.09} \mathrm{~s}$ ), similar to events in the control experiment, and a long duration population not seen in the control experiment (duration $\geq 0.1 \mathrm{~s}$, mean $10^{0.43 \pm 0.03} \mathrm{~s}$ ) (Figures 3B,C, blue triangles). The shorter duration population $(<0.1 \mathrm{~s})$ appeared consistent with RNA(hairpin) absent $\phi 29$ DNAP and was statistically indistinguishable from the event population seen in the control ( $p$-value $<0.66$, two-tailed $t$-test). The longer duration population $(\geq 0.1 \mathrm{~s})$ was longer in mean duration and was statistically different from the population seen in the control ( $p$-value $<0.0001$, two-tailed $t$-test). This suggested that these long duration events were the result of $\phi 29$ DNAP binding the adapted RNA substrate and slowing strand translocation through the nanopore.

We included two abasic residues ( $1^{\prime}-\mathrm{H}$ deoxyribose) in the adapter strands near the ligation junctions with the RNA to act as indicators of strand translocation (see Figure 3A). Abasic residues have been shown to cause distinctive high current states that are apparent during enzyme-controlled translocation of oligonucleotides through the $\alpha$-HL pore (Gyarfas et al., 2009; Lieberman et al., 2010). Because the abasic residues in the adapter (subsequently referred to as a "dual-abasic adapter") flank the RNA insert, they should translocate through the nanopore before and after the RNA insert (Figure 4A). This should produce an ionic current trace with high current states bracketing an intervening region and indicate strand translocation occurred in a linear conformation. Further, the intervening region would correspond

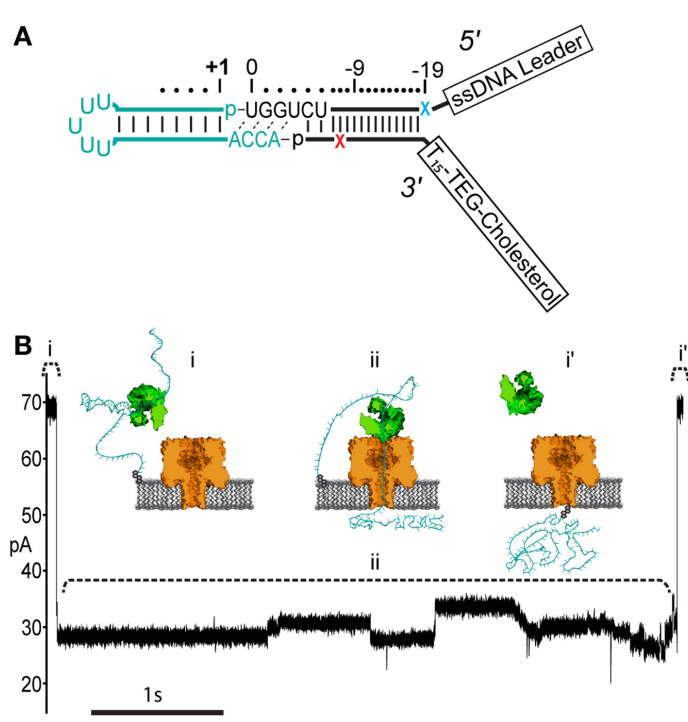

FIGURE 3 | Nanopore capture of adapted RNA complexed with non-catalytic $\phi \mathbf{2 9}$ DNAP. (A) Schematic of the synthetic RNA(hairpin) construct (cyan) covalently attached to the adapter (black). This simple RNA hairpin is composed of a synthetic copy of the E. coli tRNA ${ }^{\text {fMet }}$ acceptor stem linked by a five uracil loop. This RNA was enzymatically ligated to the adapter as described previously (see Materials and Methods). Colored X's indicate $5^{\prime}$ (blue) and $3^{\prime}$ (red) abasic residues in the adapter strands. (B) Representative ionic current trace during capture of an adapted RNA hairpin complexed with $\phi 29$ DNAP. $\$ 29$ DNAP $(75 \mathrm{nM})$ and adapted RNA $(0.5 \mathrm{nM})$ were added to cis side compartment which contained nanopore

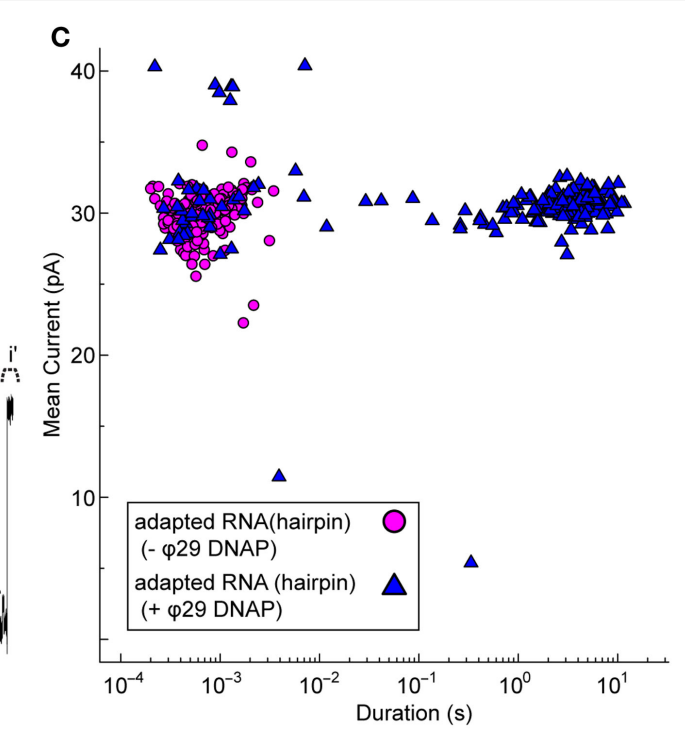

buffer absent $\mathrm{Mg}^{2+}$ and amended with $1 \mathrm{mM}$ EDTA (see Materials and Methods). The cartoons above the ionic current trace represent proposed steps during $\phi 29$ DNAP-controlled translocation: (i) open channel prior to capture of the adapted RNA: $\phi 29$ DNAP complex; (ii) nanopore capture and translocation of adapted RNA bound to $\phi 29$ DNAP; (i') return to open channel current when the RNA clears the pore into the trans compartment. Scale bar indicates $1 \mathrm{~s}$. (C) Mean ionic current versus duration for adapted RNA(hairpin)-dependent blockades absent (magenta circles) or present (blue triangles) $\phi 29$ DNAP. Approximately 200 events are shown for each condition from a representative single experiment. 

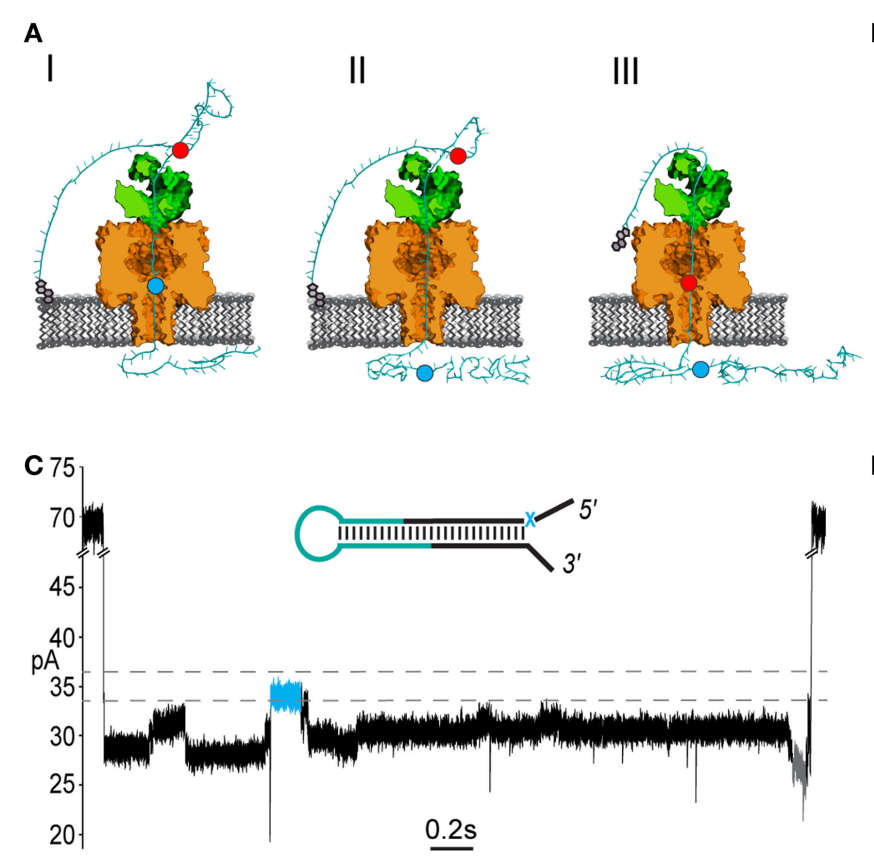

FIGURE 4 | End-to-end translocation of adapted RNA(hairpin) constructs through the $\boldsymbol{\alpha}-\mathbf{H L}$ nanopore. (A) A cartoon model showing capture and translocation of an adapted RNA(hairpin) complexed with $\phi 29$ DNAP. The adapted RNA(hairpin) substrate is described in Figure 3. (I) Following capture of the $5^{\prime}$ end of the adapter strand, the polynucleotide translocates through $\alpha-H L$ until the leading $5^{\prime}$ abasic residue (blue circle) reaches pore limiting aperture. (II) Translocation continues through the RNA portion of the adapted molecule. (III) The trailing $3^{\prime}$ abasic residue (red circle) reaches the pore limiting aperture after translocation of the RNA(hairpin). (B) A nanopore ionic current trace during translocation of an adapted RNA(hairpin) (inset), which corresponding to the cartoon
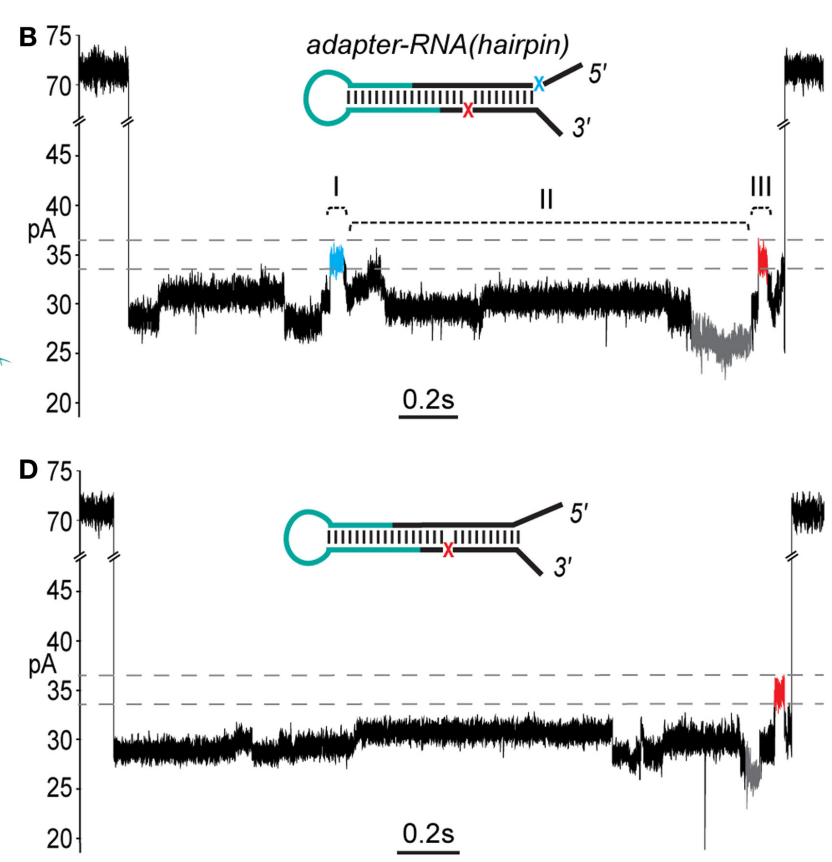

in (A). The event displays two high current marker regions characteristic of the $5^{\prime}$ abasic residue (blue segment) and $3^{\prime}$ abasic residue (red segment) built into the adapter. Translocation events that contained both leading and trailing markers were observed in $\sim 27 \%$ of events with durations exceeding $1 \mathrm{~s}$. The marker ionic current level was 33.5-36.5 pA (dashed gray lines) under the experimental conditions used (see Materials and Methods). Scale bar indicates $0.2 \mathrm{~s}$. (C) A nanopore ionic current trace during translocation of an adapted RNA(hairpin) (inset) bearing only the $5^{\prime}$ abasic residue ( $5^{\prime}$ mono-abasic adapted $\left.\mathrm{RNA}\right)$. (D) A nanopore ionic current trace during translocation of an adapted RNA(hairpin) (inset) bearing only the $3^{\prime}$ abasic residue ( $3^{\prime}$ mono-abasic adapted RNA). to the adapted RNA(hairpin) insert traversing the pore. As seen with DNA during $\$ 29$ DNAP-mediated translocation, the individual ribonucleobases of the extended chain should directly modulate the ionic current during translocation through the $\alpha$-HL pore, resulting in a reproducible pattern of ionic current states (Cherf et al., 2012).

As predicted, translocation of dual-abasic adapted RNA(hairpin) constructs bound to $\phi 29$ DNAP resulted in blockade events containing two distinct ionic current states in the range of 33.5-36.5 pA that flank a reproducible pattern of ionic current (Figure 4B; Figure S1 in Supplementary Material). This suggested that the adapted RNA(hairpin) translocated completely through the nanopore, and that $\phi 29$ DNAP acted as a passive molecular brake for both the DNA and RNA portions of the chimeric molecule.

\section{The Ionic Current Pattern Produced by $5^{\prime}$ and $3^{\prime}$ Abasic Residues Establishes the Direction of Translocation and Indicates Complete Strand Translocation}

To establish the direction that $\phi 29$ DNAP-bound strands translocated through the nanopore, it was necessary to assign each of the observed high current states to either the $5^{\prime}$ or $3^{\prime}$ abasic residue. To do this, we synthesized adapters that contained only one of the $5^{\prime}$ or $3^{\prime}$ abasic residues. These "mono-abasic adapters" were ligated to the RNA(hairpin) substrate (see Figure 3A, cyan).

Substrates bearing the $5^{\prime}$ mono-abasic adapter typically produced events containing a single high current state (33.5-36.5 pA) near the beginning of the event (Figure 4C). Substrates bearing the $3^{\prime}$ mono-abasic adapter caused a similar high current state near the end of the event, which was preceded by a low current state $(<=26.5 \mathrm{pA})$ (Figure 4D).

These observations provided an ionic current model for complete translocation of the dual abasic-adapted RNA(hairpin) in the $5^{\prime}$-to- $3^{\prime}$ direction. That is, the $5^{\prime}$ abasic residue caused the first high current state, which was followed by the intervening current region corresponding to the RNA, which includes the low current state. This is followed by the high current state proximal to the end of the event that is caused by the $3^{\prime}$ abasic residue.

Using this model, we quantified the frequency of the leading and trailing high current states (henceforth referred to as leading and trailing markers) in events $>1 \mathrm{~s}$ duration. Translocation of the dual-abasic adapted RNA resulted in $27.4 \%$ of events (102 of 372 ) that contained both the leading marker and trailing marker separated by a region containing a low current segment (Table 1). An additional $43 \%$ of events were classified as containing only a leading marker and $6.7 \%$ were classified as containing only a 
TABLE 1 | RNA(hairpin) nanopore translocation events classified by detection of leading and trailing high current markers

\begin{tabular}{|c|c|c|c|c|c|}
\hline Substrate & Events & $\begin{array}{l}\text { Leading and trailing } \\
\text { high current observed }^{\mathrm{a}}\end{array}$ & $\begin{array}{l}\text { Leading high } \\
\text { current observed }^{\text {b }}\end{array}$ & $\begin{array}{c}\text { Trailing high } \\
\text { current observed }^{\mathrm{c}}\end{array}$ & Other events ${ }^{d}$ \\
\hline RNA(hairpin) (dual-abasic adapter) & 372 & $102(27.4 \%)$ & $160(43.0 \%)$ & $25(6.7 \%)$ & 85 (22.9\%) \\
\hline RNA(hairpin) (5' mono-abasic adapter) & 287 & $3(1.0 \%)$ & $185(64.5 \%)$ & $10(3.5 \%)$ & $89(31.0 \%)$ \\
\hline RNA(hairpin) (3' mono-abasic adapter) & 285 & $3(1.1 \%)$ & $4(1.4 \%)$ & $108(37.9 \%)$ & $170(59.6 \%)$ \\
\hline
\end{tabular}

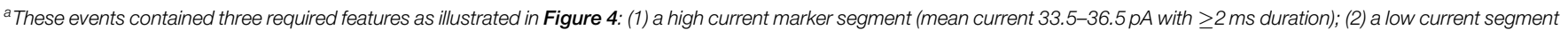
(mean current $=<26.5 \mathrm{pA}$ with $\geq 10 \mathrm{~ms})$; (3) a second high current segment proximal to the termination of the event.

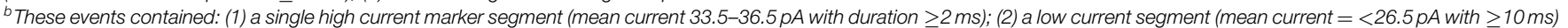
that always followed the high current marker.

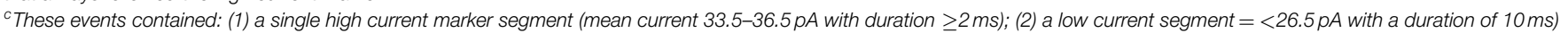
that always preceded the high current marker segment.

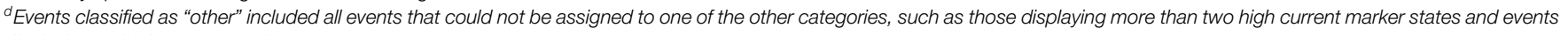
displaying no high current marker segments.

All events were $\geq 1$ s duration.

trailing marker. The disparity in frequency between leading and trailing markers suggested that the $3^{\prime}$ end of the strand was more difficult to resolve.

The less frequent observation of the trailing marker relative to the leading marker suggested two possible explanations: either the $3^{\prime}$ abasic was absent from many strands, or that the translocation rate increased in the region of the $3^{\prime}$ abasic. In experiments with dual-abasic molecules, where both $5^{\prime}$ and $3^{\prime}$ abasic markers were resolved, the duration of the $3^{\prime}$ marker is notably shorter (Figure 4B; Figure S1A in Supplementary Material), supporting the latter hypothesis. Furthermore, in experiments with constructs containing mono-abasic adapters, we observed the same trends: strands bearing the $5^{\prime}$ mono-abasic adapter produced events with a single high current region $64.5 \%$ of the time with notably longer duration (Table 1 and Figure 4C; Figure S1B in Supplementary Material), and strands bearing the $3^{\prime}$ mono-abasic adapter produced a smaller fraction of events (37.9\%) with a single highcurrent marker of shorter duration (Table 1 and Figure 4D; Figure $\mathrm{S} 1 \mathrm{C}$ in Supplementary Material). This suggests that the translocation rate of the molecule increases after $\phi 29$ DNAP unzips the double stranded region. This would produce shorter duration ionic current signatures at the $3^{\prime}$ end. We reasoned that these shorter duration $3^{\prime}$ trailing markers were frequently unresolved at the measurement timescale used by our apparatus.

The result of the experiments using mono-abasic markers is consistent with $5^{\prime}$-to- $3^{\prime}$ traversal of adapted RNA through the $\alpha$ HL pore when bound to $\$ 29$ DNAP. By design, the $3^{\prime}$ end of adapted RNA would be retained in or at the lipid bilayer. This would preferentially leave the $5^{\prime}$ end of adapted RNA molecules available to initiate threading through the pore and act as the loading site for $\phi 29$ DNAP. By experiment, when a single abasic residue was present only on the leading strand of the adapter $\left(5^{\prime}\right)$, we observed a single long-duration high current marker toward the beginning of $\phi 29$ DNAP-bound translocation events. Conversely, inclusion of a single abasic residue only on the trailing strand of the adapter $\left(3^{\prime}\right)$ produced a single short-duration high current marker toward the end of $\phi 29$ DNAP-bound translocation events. Presence of both abasic residues in the adapter resulted in $\sim 27 \%$ of $\phi 29$ DNAP-bound translocation events containing two high current markers. Taken together, these results support initiation of the $\phi 29$ DNAP-bound strand threading through the nanopore from the $5^{\prime}$ end, followed by the RNA portion, and terminating with the $3^{\prime}$ end of strand passing through and exiting the pore. A $5^{\prime}$-to- $3^{\prime}$ voltage-driven "unzipping" process was previously observed for $\phi 29$ DNAP under non-enzymatic conditions on DNA (Lieberman et al., 2010). We conclude from the results presented here that: $\phi 29$ DNAP is being driven in the $5^{\prime}-3^{\prime}$ direction, it unzips the dual-abasic adapted RNA(hairpin) strand, and in events where both markers were observed, the strand is translocated through the nanopore in its entirety. Further, these markers provide approximate boundaries of the RNA-dependent portion of the nanopore signal.

\section{E. coli tRNA ${ }^{\mathrm{fMet}}$ and tRNA ${ }^{\text {Lys }}$ can be Classified Based on Their Nanopore Current Signals}

If the ionic current segment flanked by the high current markers contains the RNA-dependent portion of nanopore signal, then that region should differentiate tRNA species. Further, a tRNAspecific change seen in the putative RNA-dependent region, when bordered by the adapter-dependent marker regions, would be evidence that the adapted tRNA translocate entirely through the nanopore. We used the $\phi 29$ DNAP-mediated braking method, as had been done with the RNA(hairpin) substrate, to improve temporal resolution of adapted tRNA. For this experiment, we selected two well-characterized tRNA species for nanopore analysis, $E$. coli $\mathrm{tRNA}^{\mathrm{fMet}}$ and $E$. coli $\mathrm{tRNA}^{\mathrm{Lys}}$. These tRNAs exist in the $E$. coli genome as either a single isoform (tRNA ${ }^{\text {Lys }}$ ) or as two isoforms that differ by only a single nucleotide $\left(\mathrm{tRNA}^{\mathrm{fMet}}\right.$ ) (RajBhandary, 1994). Additionally, tRNA ${ }^{\text {Lys }}$ and tRNA ${ }^{\text {fMet }}$ have similar lengths (76 and $77 \mathrm{nt}$, respectively), but have significantly different nucleotide compositions (50.0 and 64.9\% G-C content, respectively), and would be expected to generate different ionic current signals.

Experiments with the adapted tRNA ${ }^{\text {fMet }}$ substrate produced 85 events that contained the leading and trailing markers bracketing an extended intervening current region (17.6\% of 481 total events) (Figure 5A; Figure S2 in Supplementary Material). Experiments with the adapted tRNA ${ }^{\text {Lys }}$ substrate produced 77 events that contained the leading and trailing markers also bracketing an extended intervening current region (22.3\% of 348 total events) (Figure 5B; Figure S2 in Supplementary Material). As suggested by the results with the adapted RNA(hairpin) substrate, these events were presumed to result from complete translocation of the adapted tRNA through the nanopore. These events were selected for further analysis of tRNA-specific ionic current signal. 


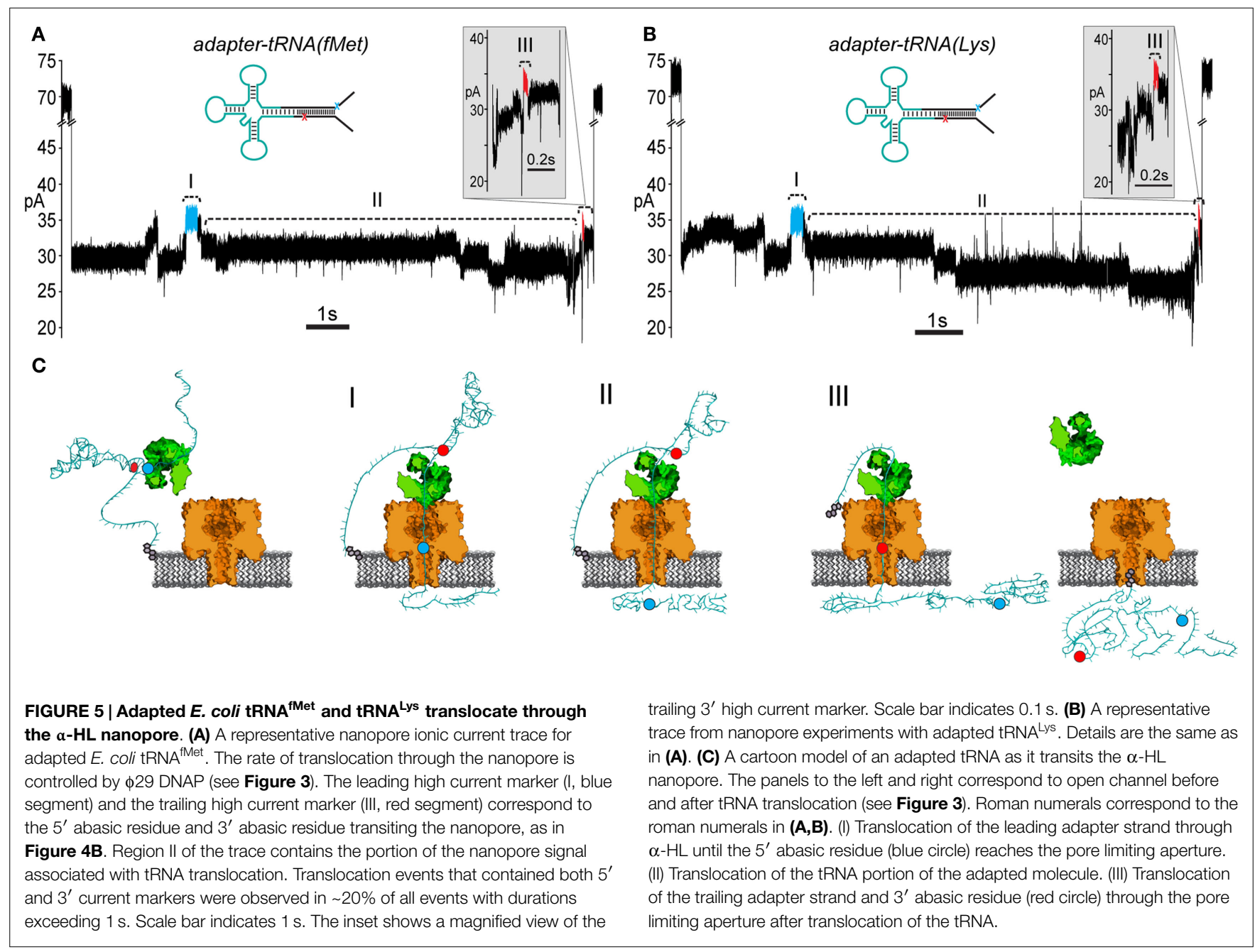

To test the putative RNA-dependent region for tRNA-specific signal (see Figures 5A,B, region II), we segmented the ionic current signal from each translocation event into regions I through III. As was the case for the RNA(hairpin) substrate (see Figure 4), regions I and III included the leading and trailing markers, respectively, which corresponded to the $5^{\prime}$ and $3^{\prime}$ abasic residues of the adapter translocating through the nanopore. These served as control regions for our analysis (Figure 5C). Bracketed by these markers, region II was expected to change based on the identity of the tRNA. Initial inspection of ionic current parameters, mean current, and dwell time suggested that Region II provided the best discrimination between tRNA ${ }^{\mathrm{fMet}}$ and $\mathrm{tRNA}^{\mathrm{Lys}}$ (Figure 6). As expected, mean current and duration for regions I and III did not appear to differ between RNA $^{\mathrm{fMet}}$ and tRNA ${ }^{\mathrm{Lys}}$.

To quantitatively assess the influence of tRNA type on ionic current, we analyzed the data in Figure 6 using a soft-margin support vector machine (SVM) (Cortes and Vapnik, 1995). A SVM was used to quantify the discrimination between the two tRNA species using their ionic current parameters in each of the three regions. The SVM produced an optimal linear decision boundary for the event log-durations and mean currents plotted in Figure 6. We used 5-way cross validation to calculate classification accuracy (mean and SD) of the boundaries produced for each of the regions (see Materials and Methods). Regions I and III provided discrimination between the two tRNA only slightly better than chance at $60.0 \pm 6.9$ and $59.4 \pm 7.0 \%$ accuracy, respectively. In contrast, the putative tRNA dependent region II provided a classification accuracy of $87.2 \pm 5.3 \%$. This result demonstrated quantitatively that tRNA contributed to the nanopore signal in region II. Further, because the adapter-dependent regions I and III flanked region II, as they do in the adapted tRNA oligonucleotide strand, we concluded that adapted tRNA translocated completely through $\alpha-\mathrm{HL}$ as an unfolded, linear strand in these instances.

\section{Discussion}

In summary, we have shown that individual biological tRNA molecules can be unfolded and translocated through a nanopore as linear strands. To facilitate this, we developed a double-stranded oligonucleotide adapter that could be enzymatically ligated to biological tRNA. The two strands of the adapter acted to locally concentrate adapted tRNA at the bilayer and to initiate strand threading through the nanopore. Using $\$ 29$ DNAP under noncatalytic conditions, we were able to slow strand translocation through the pore. This allowed us to observe adapted tRNA translocating $5^{\prime}$-to- $3^{\prime}$ through the pore as a linear strand when 

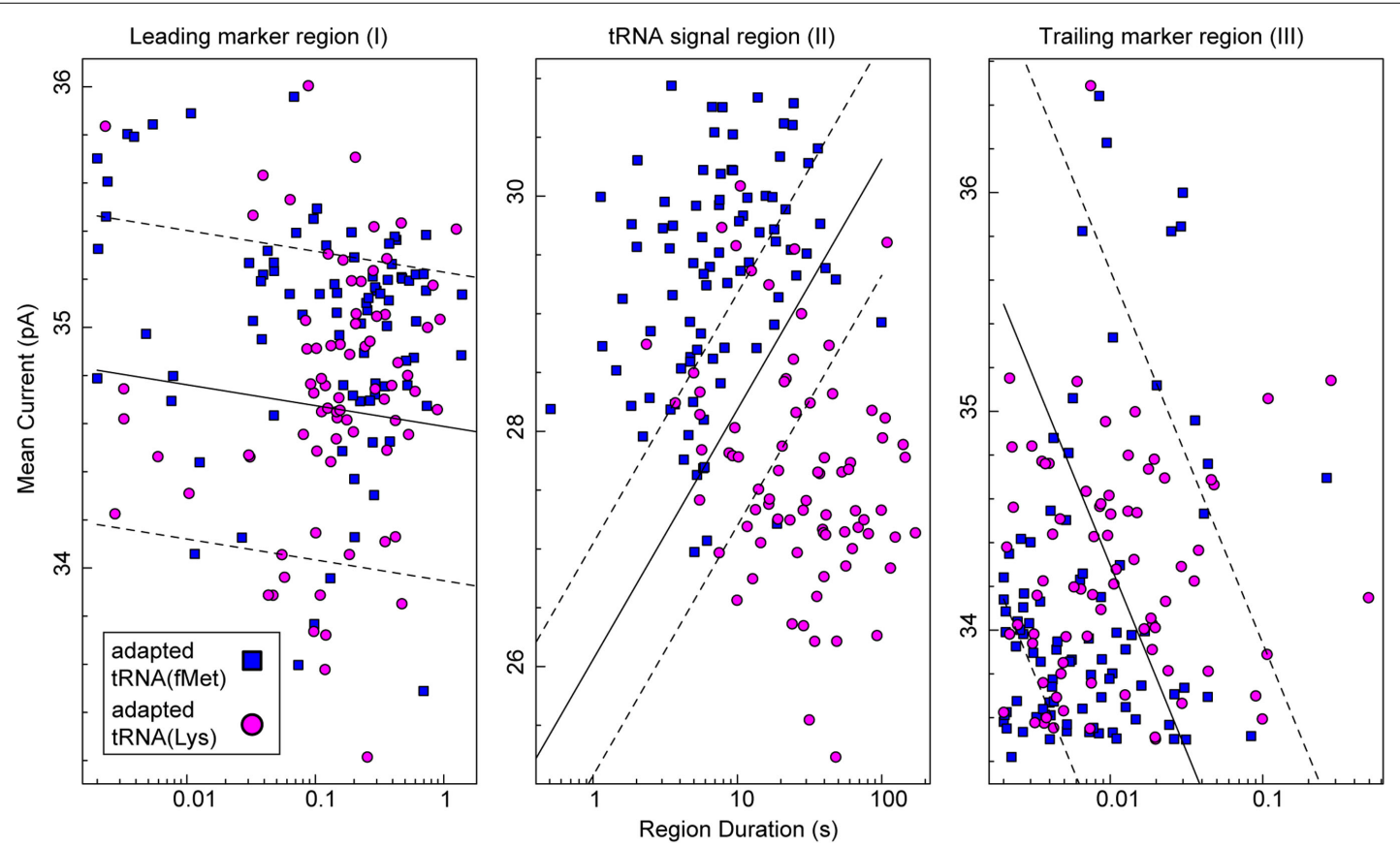

FIGURE 6 | Classification of tRNA molecules using duration and mean ionic for regions I-III of adapted tRNA translocation events. We collected complete ionic current events (approximately 80 for each class) from experiments $(n \geq 5)$ using adapted $E$. coli tRNA ${ }^{\text {fMet }}$ (blue squares) or adapted tRNA ${ }^{\text {Lys }}$ (magenta circles). In our model (Figure 5C), regions I and III correspond to the common leading and trailing marker regions, respectively, and region II corresponds to the intervening RNA-dependent portion of the signal. The $x$-axis is the duration of a given region and the $y$-axis is mean current for that region. In each panel, the solid black line is a semi-logarithmic decision boundary established for that region using a soft-margin Support Vector Machine (SVM) (see Materials and Methods). SVM margins are shown as dashed lines. The associated classification accuracy for region II was $87.2 \pm 5.3 \%$. The associated classification accuracies for regions I and III were $60.0 \pm 6.9$ and $59.4 \pm 7.0 \%$, respectively. The SVM classification accuracy (mean and SD) was established using fivefold validation (see Materials and Methods). Data for the two tRNA species were collected separately from at least five independent nanopore experiments. bound by $\phi 29$ DNAP. Finally, as evidence that tRNA influenced ionic current during translocation, we show that $E$. coli RNA $^{\mathrm{fMet}}$ and $\mathrm{tRNA}^{\text {Lys }}$ produced differentiable ionic current signals.

Linear translocation of biological tRNA through the nanopore is a first step toward single-molecule direct sequence analysis of tRNA. Full implementation of a nanopore-based RNA sequencing method will require the following improvements: (1) increased sensitivity of the nanopore: for example, pores derived from mycobacterial species provide nucleotide-level sensitivity for DNA sequencing (Manrao et al., 2012). (2) Regulation of tRNA movement in single nucleotide steps: this may be accomplished by coupling an active molecular motor to translocate tRNA, as has been accomplished for DNA sequencing using $\$ 29$ DNAP in a catalytic mode (Laszlo et al., 2014). (3) Correlation of ionic current states with nucleotide identity: this has been achieved for canonical DNA bases and for various cytosine modifications (Manrao et al., 2012; Schreiber et al., 2013; Wescoe et al., 2014). (4) Increased throughput: for example, use of multi-channel nanopore devices will allow for reading tens of thousands of individual tRNA molecules.

Mitochondrial tRNA (mt.tRNA) genes comprise $<10 \%$ of the mitochondrial genome $(16,569 \mathrm{bp})$, yet $43 \%(245 / 571)$ of the currently known pathogenic mutations cataloged in MITOMAP are found in these genes (Brandon et al., 2005; Putz et al., 2007; Ruiz-Pesini et al., 2007). The lack of a direct view of transcribed mt.tRNA prevents use of sequence, modification state, and tRNA abundance as a facile diagnostic in mitochondrial pathology. We expect that a mature nanopore-based method for directly sequencing individual tRNA molecules will yield both canonical base identity and nucleotide modification states along entire strands. This will help advance our understanding of tRNA biology and the diagnosis of mitochondrial pathology as we fully appreciate the role of tRNA in human disease.

\section{Acknowledgments}

Oxford Nanopore Technologies provided funding to support this work (SC20130149), $\alpha$-HL protein, initial study of $\phi 29$ DNAPmediated braking of RNA, and the cholesterol tagging strategy. Jacob Schreiber developed software for nanopore event detection and visualization. Dr. Todd Lowe provided S. cerevisiae tRNA ${ }^{\text {Phe }}$ and Dr. Harry Noller provided E. coli tRNA ${ }^{\text {fMet }}$ and tRNA ${ }^{\text {Lys }}$.

\section{Supplementary Material}

The Supplementary Material for this article can be found online at http://journal.frontiersin.org/article/10.3389/fbioe.2015.00091 


\section{References}

Akeson, M., Branton, D., Kasianowicz, J. J., Brandin, E., and Deamer, D. W. (1999). Microsecond time-scale discrimination among polycytidylic acid, polyadenylic acid, and polyuridylic acid as homopolymers or as segments within single RNA molecules. Biophys. J. 77, 3227-3233. doi:10.1016/S0006-3495(99)77153-5

Ayub, M., and Bayley, H. (2012). Individual RNA base recognition in immobilized oligonucleotides using a protein nanopore. Nano Lett. 12, 5637-5643. doi:10. 1021/nl3027873

Bernick, D. L., Dennis, P. P., Höchsmann, M., and Lowe, T. M. (2012). Discovery of pyrobaculum small RNA families with atypical pseudouridine guide RNA features. RNA 18, 402-411. doi:10.1261/rna.031385.111

Brandon, M. C., Lott, M. T., Nguyen, K. C., Spolim, S., Navathe, S. B., Baldi, P., et al. (2005). MITOMAP: a human mitochondrial genome database - 2004 update. Nucleic Acids Res. 33, D611-D613. doi:10.1093/nar/gki079

Chan, C. T. Y., Dyavaiah, M., DeMott, M. S., Taghizadeh, K., Dedon, P. C., and Begley, T. J. (2010). A quantitative systems approach reveals dynamic control of tRNA modifications during cellular stress. PLoS Genet. 6:e1001247. doi:10.1186/ 1477-3155-11-8

Chan, C. T. Y., Pang, Y. L. J., Deng, W., Babu, I. R., Dyavaiah, M., Begley, T. J., et al. (2012). Reprogramming of tRNA modifications controls the oxidative stress response by codon-biased translation of proteins. Nat Commun 3, 937. doi:10.1038/ncomms1938

Chan, P. P., Cozen, A. E., and Lowe, T. M. (2011). Discovery of permuted and recently split transfer RNAs in Archaea. Genome Biol. 12, R38. doi:10.1186/ gb-2011-12-4-r38

Cherf, G. M., Lieberman, K. R., Rashid, H., Lam, C. E., Karplus, K., and Akeson, M. (2012). Automated forward and reverse ratcheting of DNA in a nanopore at 5 precision. Nat. Biotechnol. 30, 344-348. doi:10.1038/nbt.2147

Cortes, C., and Vapnik, V. (1995). Support-vector networks. Mach Learn 20, 273-297. doi:10.1007/BF00994018

Deamer, D. W., and Branton, D. (2002). Characterization of nucleic acids by nanopore analysis. Acc. Chem. Res. 35, 817-825. doi:10.1021/ar000138m

Dittmar, K. A., Goodenbour, J. M., and Pan, T. (2006). Tissue-specific differences in human transfer RNA expression. PLoS Genet. 2:e221. doi:10.1371/journal.pgen. 0020221

Dominissini, D., Moshitch-Moshkovitz, S., Schwartz, S., Salmon-Divon, M., Ungar, L., Osenberg, S., et al. (2012). Topology of the human and mouse m6A RNA methylomes revealed by m6A-seq. Nature 485, 201-206. doi:10.1038/ nature 11112

Gustilo, E. M., Vendeix, F. A., and Agris, P. F. (2008). tRNA's modifications bring order to gene expression. Curr. Opin. Microbiol. 11, 134-140. doi:10.1016/j.mib. 2008.02.003

Gyarfas, B., Olasagasti, F., Benner, S., Garalde, D. R., Lieberman, K., and Akeson, M. (2009). Mapping the position of DNA polymerase-bound DNA templates in a nanopore at $5 \AA$ resolution. ACS Nano 3, 1457-1466. doi:10.1021/ nn900303g

Hiley, S., Jackman, J., Babak, T., Trochesset, M., Morris, Q., Phizicky, E. M., et al. (2005). Detection and discovery of RNA modifications using microarrays. Nucleic Acids Res. 33, e2. doi:10.1093/nar/gni002

Karatzoglou, A., Smola, A., Hornik, K., and Zeileis, A. (2004). Kernlab - an S4 package for kernel methods in R. J. Stat. Softw. 11. Available at: http://www. jstatsoft.org/v11/i09/paper

Kasianowicz, J. J., Brandin, E., Branton, D., and Deamer, D. W. (1996). Characterization of individual polynucleotide molecules using a membrane channel. Proc Natl Acad Sci U.S.A. 93, 13770-13773. doi:10.1073/pnas.93.24.13770

Kirino, Y., Yasukawa, T., Ohta, S., Akira, S., Ishihara, K., Watanabe, K., et al. (2004). Codon-specific translational defect caused by a wobble modification deficiency in mutant tRNA from a human mitochondrial disease. Proc Natl Acad Sci U.S.A. 101, 15070-15075. doi:10.1073/pnas.0405173101

Laszlo, A. H., Derrington, I. M., Brinkerhoff, H., Langford, K. W., Nova, I. C., Samson, J. M., et al. (2013). Detection and mapping of 5-methylcytosine and 5-hydroxymethylcytosine with nanopore MspA. Proc Natl Acad Sci U.S.A. 110, 18904-18909. doi:10.1073/pnas.1310240110

Laszlo, A. H., Derrington, I. M., Ross, B. C., Brinkerhoff, H., Adey, A., Nova, I. C., et al. (2014). Decoding long nanopore sequencing reads of natural DNA. Nat. Biotechnol. 32, 829-833. doi:10.1038/nbt.2950

Lieberman, K. R., Cherf, G. M., Doody, M. J., Olasagasti, F., Kolodji, Y., and Akeson, M. (2010). Processive replication of single DNA molecules in a nanopore catalyzed by $\phi 29$ DNA polymerase. J. Am. Chem. Soc. 132, 17961-17972. doi:10. $1021 /$ ja 1087612

Manrao, E. A., Derrington, I. M., Laszlo, A. H., Langford, K. W., Hopper, M. K., Gillgren, N., et al. (2012). Reading DNA at single-nucleotide resolution with a mutant MspA nanopore and $\$ 29$ DNA polymerase. Nat. Biotechnol. 30, 349-353. doi:10.1038/nbt.2171

Merino, E. J., Wilkinson, K. A., Coughlan, J. L., and Weeks, K. M. (2005). Selective $2^{\prime}$-hydroxyl acylation analyzed by primer extension (SHAPE): quantitative RNA structure analysis at single nucleotide resolution. J. Am. Chem. Soc. 127, 4223-4231. doi:10.1021/ja043822v

Motorin, Y., and Helm, M. (2010). tRNA stabilization by modified nucleotides. Biochemistry 49, 4934-4944. doi:10.1021/bi100408z

Omer, A. D., Lowe, T. M., Russell, A. G., Ebhardt, H., Eddy, S., and Dennis, P. (2000). Homologs of small nucleolar RNAs in Archaea. Science 288, 517-522. doi:10.1126/science.288.5465.517

Phizicky, E. M., and Hopper, A. K. (2010). tRNA biology charges to the front. Genes Dev. 24, 1832-1860. doi:10.1101/gad.1956510

Putz, J., Dupuis, B., Sissler, M., and Florentz, C. (2007). Mamit-tRNA, a database of mammalian mitochondrial tRNA primary and secondary structures. RNA 13, 1184-1190. doi:10.1261/rna.588407

Raina, M., and Ibba, M. (2014). tRNAs as regulators of biological processes. Front. Genet. 5:171. doi:10.3389/fgene.2014.00171

RajBhandary, U. (1994). Initiator transfer RNAs. J. Bacteriol. 176, 547-552.

Ruiz-Pesini, E., Lott, M. T., Procaccio, V., Poole, J. C., Brandon, M. C., Mishmar, D., et al. (2007). An enhanced MITOMAP with a global mtDNA mutational phylogeny. Nucleic Acids Res. 35, D823-D828. doi:10.1093/nar/gkl927

Saikia, M., Fu, Y., Pavon-Eternod, M., He, C., and Pan, T. (2010). Genomewide analysis of N1-methyl-adenosine modification in human tRNAs. RNA 16, 1317-1327. doi:10.1261/rna.2057810

Schaefer, M., Pollex, T., Hanna, K., and Lyko, F. (2009). RNA cytosine methylation analysis by bisulfite sequencing. Nucleic Acids Res. 37, e12. doi:10.1093/nar/ gkn954

Schreiber, J., Wescoe, Z. L., Abu-Shumays, R., Vivian, J. T., Baatar, B., Karplus, K., et al. (2013). Error rates for nanopore discrimination among cytosine, methylcytosine, and hydroxymethylcytosine along individual DNA strands. Proc Natl Acad Sci U.S.A. 110, 18910-18915. doi:10.1073/pnas.1310615110

Serebrov, V., Clarke, R. J., Gross, H. J., and Kisselev, L. (2001). $\mathrm{Mg}^{2+}$-induced tRNA folding. Biochemistry 40, 6688-6698. doi:10.1021/bi002241p

Stein, A., and Crothers, D. M. (1976). Conformational changes of transfer RNA. The role of magnesium(II). Biochemistry 15, 160-168. doi:10.1021/bi00646a025

Suzuki, T., Nagao, A., and Suzuki, T. (2011). Human mitochondrial diseases caused by lack of taurine modification in mitochondrial tRNAs. Wiley Interdiscip Rev RNA 2, 376-386. doi:10.1002/wrna.65

Vilfan, I. D., Tsai, Y.-C., Clark, T. A., Wegener, J., Dai, Q., Yi, C., et al. (2013). Analysis of RNA base modification and structural rearrangement by singlemolecule real-time detection of reverse transcription. J. Nanobiotechnology. 11, 8. doi:10.1186/1477-3155-11-8

Wanunu, M., Dadosh, T., Ray, V., Jin, J., McReynolds, L., and Drndic, M. (2010). Rapid electronic detection of probe-specific microRNAs using thin nanopore sensors. Nat. Nanotechnol. 5, 807-814. doi:10.1038/nnano.2010.202

Wescoe, Z. L., Schreiber, J., and Akeson, M. (2014). Nanopores discriminate among five C5-cytosine variants in DNA. J. Am. Chem. Soc. 136, 16582-16587. doi:10. $1021 / \mathrm{ja} 508527 \mathrm{~b}$

Yasukawa, T., Suzuki, T., Ishii, N., Ohta, S., and Watanabe, K. (2001). Wobble modification defect in tRNA disturbs codon-anticodon interaction in a mitochondrial disease. ЕMBO J. 20, 4794-4802. doi:10.1093/emboj/20.17.4794

Yi, C., and Pan, T. (2011). Cellular dynamics of RNA modification. Acc. Chem. Res. 44, 1380-1388. doi:10.1021/ar200057m

Conflict of Interest Statement: The authors declare the following competing financial interest(s): Mark Akeson is a consultant to Oxford Nanopore Technologies. Patent application: SC2014_725_PRV, Molecular adapter for capture and manipulation of tRNA.

Copyright ( 2015 Smith, Abu-Shumays, Akeson and Bernick. This is an open-access article distributed under the terms of the Creative Commons Attribution License (CC $B Y$ ). The use, distribution or reproduction in other forums is permitted, provided the original author(s) or licensor are credited and that the original publication in this journal is cited, in accordance with accepted academic practice. No use, distribution or reproduction is permitted which does not comply with these terms. 